\title{
The Study of Allelopathic Effects of Taxus Chinensis Var.mairei on Different Crops
}

\author{
De-Ying Deng, Ling Wang, Yu-Xian Li* \\ College of Pharmacy, Henan University of Traditional Chinese Medicine, Zhengzhou 450008, P.R. China;
}

Li-Ping Zhu, Ke Yuan

Zhejiang Agriculture and Forestry University, Lin' an, China

*Corresponding author

\begin{abstract}
Background: In order to establish a scientific and reasonable intercropping pattern of Taxus chinensis var.mairei, allelopathic effects of the aqueous extracts from Taxus chinensis var.mairei on seed germination and seedling growth of rape (Brassica campestris L.), radish(Raphanus sativus L), soybean (Glycine max). Materials and Methods: lettuce (Lactuca sativa) were studied by the methods of bioassay. The results showed that the seed germination and seedling growth of four kinds of crop were dramatically inhibited when the aqueous extracts are in higher concentration, and the inhibition enhanced with the increase of the aqueous extract concentrations; lower concentration of the aqueous extracts promoted the seed germination and seedling growth of rape, radish and lettuce. Results: The allelopathic effect of the aqueous extracts from Taxus chinensis var.mairei underground part on different crops was stronger than that of aerial part. Perhaps Taxus chinensis var.mairei may contain some allelochemical, which interferes with seed germination and seedling growth of these plants, and the comprehensive inhibition effect were greater than the promoting effect. Conclusion: Consequently, the study suggests that rape, radish, soybean and lettuce are not suitable for intercropping with Taxus chinensis var.mairei, and hypothesizes that dicotyledonous crops are basically not suitable for intercropping with Taxus chinensis var.mairei.
\end{abstract}

KEYWORD: Taxus chinensis var.mairei, seed germination; seedling growth, allelopathy; interplanting

\section{INTRODUCTION}

The allelopathy is the advantageous or disadvantageous effect on the other plants or microorganisms in our environment brought about by the metabolic secretions of the plants or microorganisms[1-3]. In recent years, the research for the allelopathy has become one of the most important devices in forestry-protection, vermin-controling, increasing the crop yield and promoting the sustainable development of our environment[4-7].

Taxus chinensis var.mairei, also called "beautiful Taxus chinensis", is a species of Taxus chinensis genus, Taxus chinensis family in coniferopsida class. This plant is mainly distributed in the south of Yangtze River basin[8-10]. The Taxol[11-15] extracted from the Taxus chinensis var.mairei is a precious anti-cancer medicine with high efficiency and low toxicity. According to the literature $[16,17]$, in each 10t barks of Taxus chinensis, only $1 \mathrm{~kg}$ of Taxol can be extracted. As the first-class tree protected by our country, many areas are engaged in building medicinal forestry of Taxus chinensis var.mairei to replace the wild species, so that they can get more Taxol and the timber of Taxus chinensis. When they build the man-made forest of Taxus chinensis var.mairei, if they interplant other crops among the spaces of the medicinal plants, it not only can solve the problem of the contradiction between medicinal plants and farming crops but also can make full use of the natural resources, as well as to increase the economical benefits $[18,19]$.

This paper adopts the method of biological assay to explore the degree and difference of the influence of the infusion of the artificially- planted

Taxus chinensis var.mairei on the four kinds of crops. Meanwhile it makes a tentative discussion about whether it is suitable to interplant with other crops.

\section{EXPERIMENTAL}

In this experiment, the test material Taxus chinensis var.mairei, picked from the planting base of Taxus chinensis var.mairei in Cixi county, Zhejiang Province; the receptor materials are all local crops like Brassica campestris L., Raphanus sativus L, soybeans, Lactuca sativa, and their seeds are purchased from Cixi Seed Company. 
Separate the root, stem and leaves of the healthy fresh Taxus chinensis var.mairei; cut the leaves into small pieces of $2 \mathrm{~cm}$; Wash the leaves clean, and after naturally drying them, put them respectively into the distilled water about 5 times of the fresh weight of the plant; soak them in water for $48 \mathrm{~h}$ at the room temperature and then by using sucking filtration method we'll get the stock solutions of roots, stems and leaves of Taxus chinensis var.mairei; their concentration are all $200 \mathrm{~g} / \mathrm{L}$ (only the marking concentration); and finally keep them at low temperature for future use. Disinfect all the receptor seeds for $30 \mathrm{~min}$ by using $1 \% \mathrm{NaClO}$; first use running water to wash for $2 \sim 3$ times, and then use distilled water to wash them clean, and finally dry them in the open air for later use. Adopt the Culture Dish Filtration-paper method to have the seed germination experiment. Take the stock solution of water infusion of the roots, stems and leaves of Taxus chinensis var.mairei, and dilute it into the liquid with the mass concentration of 20,50, $100 \mathrm{~g} / \mathrm{L}$, and we'll get 4 concentration gradient together with the stock solution $(200 \mathrm{~g} / \mathrm{L})$; take respectively $5 \mathrm{ml}$ of the donor infusion and add them into the culture dish with 2 layers of filtration paper, and use the distilled water as the control group. Put the receptor seeds of equal size and plump grains onto the filtration paper with 50 seeds in each dish; and then place the culture dish in the incubator of constant temperature at $25^{\circ} \mathrm{C}$ to cultivate in the dark, and treat them three times each. Record one time the seed germination number every $24 \mathrm{~h}$, and the germinating standard is that the radicles or embryonal axis grow out of the seed skin for 1 2 $\mathrm{mm}$. At the same time, calculate the seed germinating rate and germinating index.

Adopt the filter paper method of the culture vessel [21] to make the biological determination on the seedlings. Pave two layers of filtration paper at the bottom of the $100 \mathrm{ml}$ beaker, and pour the water infusion of the roots, stems and leaves of Taxus chinensis var.mairei into the beaker respectively (20, $50,100,200 \mathrm{~g} / \mathrm{L}$ ), and use the distilled water as the control group. Put five pre-germinated receptor seedlings of the same growth condition into each dish and use sealing film to cover the beaker so that it can prevent water evaporation and the interplay between them. Put the small beaker into the incubator with the constant temperature at $25^{\circ} \mathrm{C}$, and cultivate in the incubator for $12 \mathrm{~h} / \mathrm{d}$, and make three times of repetition for each dish. At sixth day, determine the seedling root length, height and fresh weight. The seed germination rate $\mathrm{GR}=\Sigma \mathrm{Gt} /$ $\mathrm{T} \times 100 \%$, in this equation, $\mathrm{Gt}$ is the germination number in $\mathrm{t}$ days, and $\mathrm{T}$ is the total seed number. The seed germination index GI $=\Sigma(\mathrm{Gt} / \mathrm{Dt})$, in this equation, Gt refers to the germination number in $\mathrm{t}$ days, and Dt represents the corresponding germinating days. The allelopathic index (RI) is acquired according to Williamson Equation. Index:
$\mathrm{RI}=1-\mathrm{C} / \mathrm{T} \quad(\mathrm{T} \geq \mathrm{C}) \quad \mathrm{RI}=\mathrm{T} / \mathrm{C}-1 \quad(\mathrm{~T}<\mathrm{C})$, in this equation, $\mathrm{C}$ is the control value, $\mathrm{T}$ is the treatment value, when $\mathrm{RI}>0$, it indicates the allelopathic facilitating effect, when $\mathrm{RI}<0$, it indicates the allelopathic inhibitory effect,here, the absolute value of RI shows how strong the allelopathy is. The experiment data is analyzed by SPSS17.0, and the Graph Pad Prism is used to make the plotting software.

\section{RESULTS AND DISCUSS}

The influence on the crop seed germination rate and germination index can be seen in figure 1 6. It can be seen from figure 1 3, compared with the control group, when the root leach liquor of Taxus chinensis var.mairei is $50 \mathrm{~g} / \mathrm{L}$, except that the germinating rate of soybean seed is reduced, the seed germinating rates of all the other three crops (rape, radish, and asparagus lettuce) increased with various degrees. With the increasing of the concentration of the water infusion in the roots, stems and leaves of Taxus chinensis var.mairei, the seed germinating rates of the four crops become smaller and smaller, which shows that the high density of the water infusions of its roots, stems and leaves in Taxus chinensis var.mairei has allelopathic inhibitory effects on the seed germination of the four crops. The low-density of the water infusion of Taxus chinensis var.mairei has the weakest effects on changing the seed germinating trends of the three crops, which shows that the allelopathic effects of the allelopathic matter in the leaf part of the Taxus chinensis var.mairei are not so strong as that in the other two parts. It can be seen from figure 4 6, the water infusion in the root, stem and leaf part has almost the same effects on the seed germinating index as on the seed germinating rate.

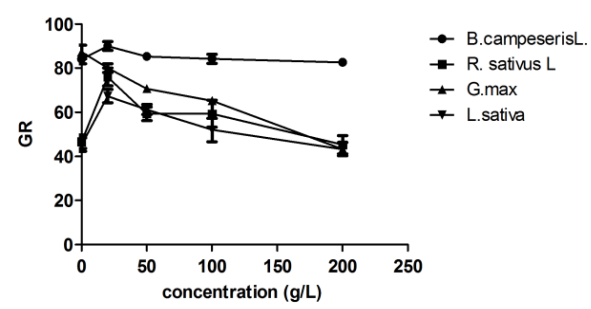

Figure 1 Effects of root aqueous extract on seed germination rate of experimental plants

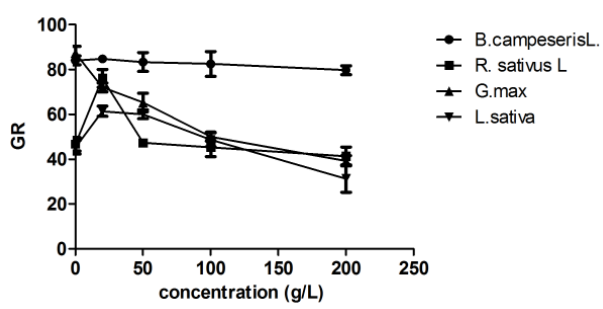

Figure 2 Effects of stem aqueous extract on seed germination rate of experimental plants 


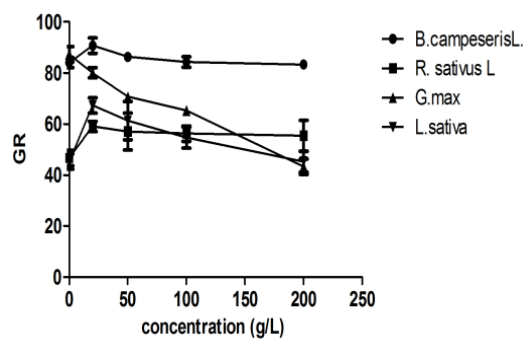

Figure 3 Effects of leaf aqueous extract on seed germination rate of experimental plants

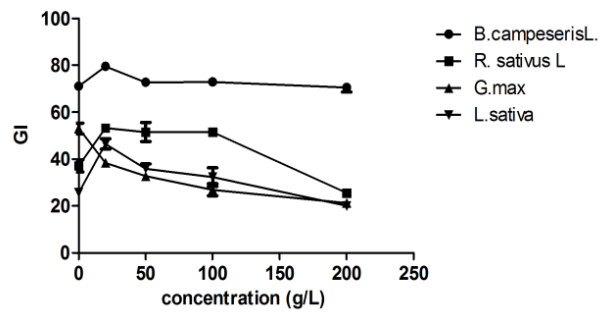

Figure 4 Effects of index aqueous extract on seed germination rate of experimental plants

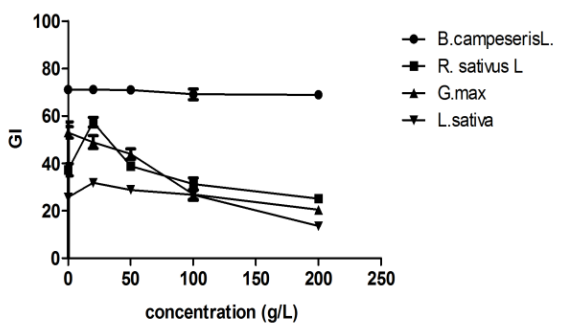

Figure 5 Effects of index aqueous extract on seed germination rate of experimental plants

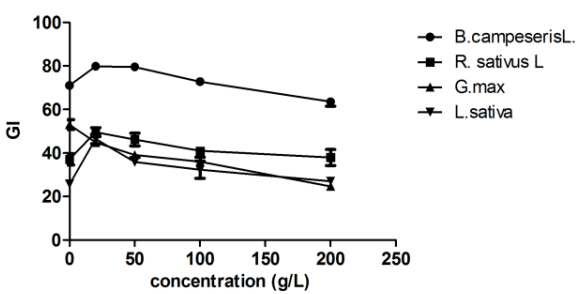

Figure 6 Effects of index aqueous extract on seed germination rate of experimental plants

The influence on the root-length and seedling height can be seen in table 1-4. It is known from table 1-4, the plant water infusions of the Taxus chinensis var.mairei show concentration effects on the growth of the root length and seedling height of the four crops. When the concentration degrees of the roots, stems and leaves of Taxus chinensis var.mairei are lower than $200 \mathrm{~g} / \mathrm{L}$, they have obvious allelopathic promoting effects on the root length and seedling height of radish; when the concentration degrees are $\geq 100 \mathrm{~g} / \mathrm{L}$, they show the inhibitory effects (in figure 2). Apart from radish, the root, stem and leaf infusion of the Taxus chinensis var.mairei all have obvious inhibitory effects on the root length and seedling height of the other three crops, and with the increasing of the concentration degree, the inhibitory effects will also increase accordingly. When the concentration of the water infusions reach $200 \mathrm{~g} / \mathrm{L}$, the roots of the soybeans begin to blacken and stop growth. The water infusions of the Taxus chinensis var.mairei have stronger inhibitory effect on the root length of the crops than that on the seedling height, and this is probably because the roots are more sensitive to its allelopathic matter. The influence on the fresh weight of the seedlings can be seen in figure7 9.

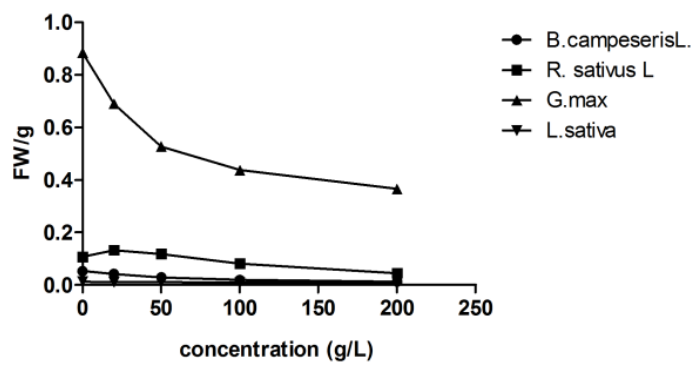

Figure 7 Effects of root aqueous extract on seedling fresh weight of experimental plants

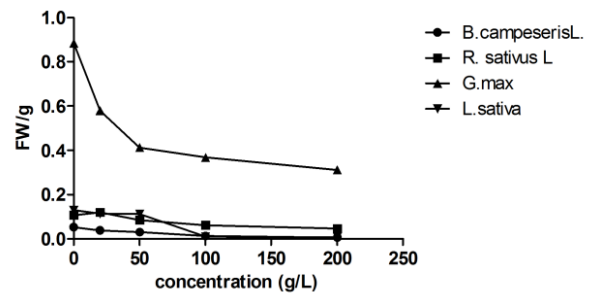

Figure 8 Effects of stem aqueous extract on seedling fresh weight of experimental plants

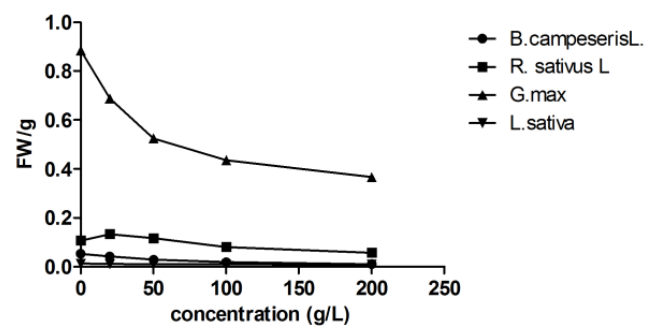

Figure 9 Effects of leaf aqueous extract on seedling fresh weight of experimental plants

From figure 7, 8, 9, we know that the water infusions of the three parts in the roots, stems and leaves of Taxus chinensis var.mairei have obvious inhibitory effects on the fresh weight of the soybean seedlings, meanwhile they have the so-called double concentration effects for the radish seedlings, that is "facilitating with lower concentration; inhibiting with higher concentration"; however, they have allelopathic inhibitory effects on the seedlings of rapes and asparagus lettuce. The water infusions of the three parts in the roots, stems and leaves of the Taxus chinensis var.mairei have inhibitory effects on the fresh weight of the seedlings of the four crops, and the inhibitory effects will increase as their concentration increase. The experiment results show that the plant water infusion of Taxus chinensis var.mairei has allelopathic inhibiting effect on the four crops. Its effects are mainly at inhibiting the seed germination, lowering the seed germinating index and hindering the growth of the seedling root length and the seedling height, as well as reducing the fresh weight of the seedlings. With the increasing of the concentration of the water infusion of Taxus chinensis var.mairei, the allelopathic inhibiting effects on the four crops will become stronger; and 
the extracting solution of the root has stronger allelopathic inhibiting effects on the four crops than those of the stems and leaves. Therefore, from the comprehensive allelopathic effects on the receptor plants, it is concluded that rapes, radish, soybeans and asparagus lettuce are all not suitable to interplant with Taxus chinensis var.mairei. Because rapes and radish are the typical small granule sensitive seeds of dicotyledons, and radish and soybean are typically large granule less sensitive seeds of dicotyledons, we can thus think that the Taxus chinensis var.mairei is not suitable to interplant with most dicotyledon plants. In the daily practice, if we want to interplant reasonably, it is proper to choose some monocotyledon seeds to interplant after observing its seed allelopathic effects. The taxanes contained in Taxus chinensis var.mairei belong to the diterpene liposoluble constituents, which can dissolved in chloroform, acetone, ethanol, but not in water. As for extracting the Taxol, we usually adopt the method of reflux extraction [21]. In the past, the medicine dregs after extracting the taxanes were thrown away as rubbish, which is a tremendous waste of resources. If the medicine dregs are developed and utilized, it will have great economic value and significance. The author of this paper proves from TLC identification that the water infusion of Taxus chinensis var.mairei contains no taxane liposoluble constituents, so it eliminates the possibility that the taxane compounds have the phytotoxin effects on the four crops. And at the same time, it suggests that in the medicine dregs of Taxus chinensis var.mairei after extracting the Taxol, there are most likely the same water-soluable allelopathic matter in the above water infusion. If we can develop these allelopathic constituents in the medicine dregs into natural herbicides, it can not only find the correct way to recycle the medicine dregs of Taxus chinensis var.mairei, but also conform to the ecocycle pattern of reusing the waste materials. However, the concrete research needs further exploration.

\section{CONCLUSION}

The water infusion of Taxus chinensis var.mairei has the inhibitory effect of various degrees on the four crops. It is thus speculated that the dicotyledon crops are basically not suitable to interplant with Taxus chinensis var.mairei; the water infusion of the Taxus chinensis var.mairei contains rich water- soluble allelochemicals, which indicates that after extracting the liposoluble constituents in the diterpene matters like paclitaxels, the medicine dregs of Taxus chinensis var.mairei may also contain such watersoluble allelochemicals; therefore, it is hopefully envisioned that in order to recycle the resources, we can use the medicine dregs of the Taxus chinensis var.mairei as raw materials of the natural herbicides.

\section{REFERENCES}

[1] Scrivanti LR, Anton AM, Zygadlo J Allelopathic potential of South American Bothriochloa species (Poaceae: Andropogoneae). Allelopathy Journal 2011; 28:189-200.

[2] Maclaren P. Chemical welfare in the forest. A Review of Allelopathy with regard to New Zealand. New Zealand Journal of Forestry 1983; 28: 75-92.

[3] Chou CH, Lee YF. Allelopathic dominance of Miscanthus transnorrisonensis in an alpine grassland community in Taiwan. Journal of Chemical Ecology 1991; 17:2267-2281.

[4] Li, J., Hong, W., Wu, C. Z., Chen, C., Song, P., Fan, H. L. Review on Allelopathy of Plants. Subtropical Agriculture Research 2007; 3 (4), 217-220.

[5] Baerson SR, Sánchez-Moreiras A, Pedrol-Bonjoch N, et al. Detoxification and transcriptome response in Arabidopsis seedlings exposed to the allelochemical benzoxazolin-2 (3H)-one. Journal of Biological Chemistry 2005; 280:21867 $-21881$.

[6] Lin DZ, Tsuzuki E, Sugimoto Y, et al. Allelopathic effects of aqueous Aloe vera leaf extracts on selected crops. Allelopathy Journal 2004; 13:67-74.

[7] Bertholdsson NO. Early vigour and allelopathy-two useful traits for enhanced barley and wheat competitiveness against weeds. Weed Research 2005; 45:94-102.

[8] Xie, Z. H., Du, L. L., Li, X. X., Xiong, Z. K. Advance on research of Taxus chinensis Var. Mairei. Monograph of Pharmacy 2009; $18: 3-5$.

[9] Li, C. F., Liu, Y., Dong, M., Shi, Q. W. Advances in studies on chemical constituents in Taxus chinensis var. mairei. Chinese Traditional and Herbal Drugs 2007; 38:1121-1132.

[10] Wang, C. T. The development and utilization of Zhejiang wild Taxus chinensis var. mairei. Journal of Anwei Agriculture Sciences 2005; 33:622-623.

[11] Stierle A, Strobel G, Stierle D. Taxol and taxane production by Taxomyces andreanae, an endophytic fungus of Pacific yew. Science 1 993; 260: 214-216.

[12] Holton RA, Kim HB, Somoza C, et al. First total synthesis of taxol. 2. Completion of the $\mathrm{C}$ and $\mathrm{D}$ rings. Journal of the American Chemical Society 1994; 116:1599-1600.

[13] Rowinsky EK, Onetto N, Canetta RM, et al. Taxol: the first of the taxanes, an important new class of antitumor agents. Seminars in oncology 1992; 19:646.

[14] Lai D, Ho KC, Hao Y, et al. Taxol resistance in breast cancer cells is mediated by the hippo pathway component TAZ and its downstream transcriptional targets Cyr61 and CTGF. Cancer Research 2011, 71:2728-2738.

[15] Wang X, Pan L, Mao N, et al. Cell-cycle synchronization reverses Taxol resistance of human ovarian cancer cell lines. Cancer cell international 2013; 13:77-82.

[16] Chen, L., Ye, X. X., Gan, Q. J. (2013). Formula experiment of Taxus plant mushroom. Edible Fungi, 2, 31-32.

[17] Kan, C. R., Cui, J. M., Cao, J. Q. Chemical composition research of North Korea's northeast Taxus after extraction of taxol production residual liquid. Chinese Traditional and Herbal Drugs 2010; 41:11-11.

[18] Li, X., Sun, J. F. The role and economic value of grass interplanting. Water Conservancy Science and Technology and Economy 2003; 9:76-76.

[19] Liao, G. H. Cultivation of high-yield, short rotation, medicinal plantation of Taxus wallichiana var. mairei. Fujian Journal of Agricultural Sciences 2009; 24:75-81.

[20] Wei Q, Zeng RS, Kong $\mathrm{CH}$, et al. The isolation and identification of allelochemicals from aerial parts of Tropical Ageratum. Acta phytoecologica sinica 1997; 21:360-366.

[21] Peng, T. H., Wang, Y. The Research of Extraction Progress of Taxol from Taxus Chinensis. Academic Periodical of Farm Products Processing 2013; 6:58-62. 